\title{
¿HAY REALMENTE UN DESACUERDO ENTRE STRAWSON Y RUSSELL RESPECTO DE LAS DESCRIPCIONES DEFINIDAS?
}

En su artículo "On Referring", Strawson esboza una teoría acerca de los actos lingüísticos, el significado, la verdad y la falsedad de lo que llama "statements", y en particular una teoría lógica acerca de los enunciados que contienen descripciones definidas. Es corriente considerar que la teoría allí expuesta pone de manifiesto algunos errores de la teoría de Russell sobre el mismo tema.

Me propongo mostrar que la teoría de Strawson es sustancialmente la misma que la de Russell, y que Strawson, y muchos de sus lectores han sido inducidos a pensar que son diferentes porque la presentación y terminología son distintas, y porque cuando se las compara no se considera una parte fundamental de la teoría de Russell. Me refiero a la distinción entre las figuraciones primarias y secundarias de las frases descriptivas en los enunciados en que aparecen. Tomando en cuenta esta distinción, cada caracterización de Strawson es el exacto reflejo de otra igualmente perfilada anteriormente por Russell.

Frente a la oración "El rey de Francia es sabio" Strawson está de acuerdo con Russell en aceptar:

1) Que la oración es significativa (y que si alguien la emitiera ahora estaría emitiendo una oración significativa).

2) Que si alguien emitiera ahora la oración, estaría formulando una aseveración verdadera sólo si, de hecho, existiera en la actualidad un único rey de Francia y dicho rey fuera sabio.

Por el contrario Strawson sostiene que Russell se equivoca cuando sostiene:

3) Que cualquiera que la emitiera ahora estaría formulando una aseveración verdadera o falsa.

4) Que parte de lo que estaría aseverando sería que en la actualidad existe un rey de Francia y sólo uno.

Esta última afirmación tiene, como mostraremos, por lo menos dos interpretaciones diferentes. En la primera, que consideraré in extenso, se sostiene que según Russell:

4') "El rey de Francia es sabio" implica "Existe un rey de Francia y sólo uno".

En cambio Strawson dirá que la primera oración no implica la segunda sino que solamente la presupone. 
Así en lugar de 4') Strawson sostendrá que:

5) "El rey de Francia es sabio" presupone pero no implica "Existe...". En lo que sigue trataré de mostrar que:

6) En el sentido en que 5) es verdadera, 5) no es incompatible con nada de lo que Russell sostiene, sino que por el contrario, se desprende de su teoría de las descripciones.

7) Que en otro sentido 5) es contradictoria y que $4^{\prime}$ ) es verdadera, y que nuevamente en esto Russell se anticipó a Strawson.

8) Que en un sentido 3) es verdadera, tanto para Russell como para Strawson, pero que en otro sentido 3) es falsa, y que de la teoría de Russell se seguiría igualmente su falsedad.

En sintesis que la teoría de Strawson no innova nada con relación a la de Russell, y que en la medida en que la de Russell estuviera equivocada, también lo estaría la de Strawson.

En consecuencia Strawson se equivoca cuando cree haber demostrado un error en la teoría de Russell.

Pasaré a continuación a exponer primero la teoría de Strawson y luego la de Russell (en los puntos pertinentes) estableciendo la correspondencia entre ambas.

\section{EL ESQUEMA TEÓRICO DE STRAWSON}

Strawson analiza las relaciones entre las oraciones, que llamaré predicativas, en las que figura una descripción definida (oraciones del tipo "El rey de Francia es sabio"), y las oraciones que llamaré existenciales (oraciones del tipo "Hay en Francia iun rey y sólo uno).

Su esquema conceptual se desarrolla en el metalenguaje de un lenguaje en que figuran tales oraciones.

En este sentido usaré ' $\mathrm{S}_{1}$ ', ' $\mathrm{S}_{2}$ ', etc., como nombres (o variables, según sea el caso) metalingüísticos de oraciones del lenguaje objeto.

Para indicar el tipo de dependencia lógica entre las oraciones predicativas y las existenciales Strawson utiliza la relación que llama de presuposición diciendo que una oración $S_{1}$ presupone a otra $S_{2}$ cuando la verdad de $S_{2}$ es condición necesaria de la verdad o la falsedad de $S_{1}$, es decir:

10) $S_{1}$ presupone $S_{2}$ si y sólo si Si $S_{1}$ es $V$ o $S_{1}$ es $F$ entonces $S_{2}$ es $V$ (de aquí en adelante ' $V$ ' abrevia 'Verdadero', y ' $F$ ' 'Falso'). Otras formas equivalentes de expresar lo mismo son:

11) $S_{1}$ presupone $S_{2}$ si y'sólo si $\left\{\begin{array}{l}\text { a) } S i S_{1} \text { es } V \text { entonces } S_{2} \text { es } V, y \\ \text { b) Si } S_{1} \text { es } F \text { entonces } S_{2} \text { es } V .\end{array}\right.$

12) $S_{1}$ presupone $S_{2}$ si y sólo si Si $S_{2}$ no es $V$ entonces $S_{1}$ no es $V$ ni F ( $S_{1}$ no es $V$ y $S_{1}$ no es $F$ ). 
Una de las tesis fundamentales de Strawson es que si $S_{1}$ es una oración predicativa y $S_{2}$ la correspondiente existencial entonces $S_{1}$ presupone $S_{2}$.

Para comprender mejor esta tesis es necesario aclarar cuándo para Strawson una oración predicativa es verdadera y cuándo es falsa.

Sea $S_{1}$ una oración predicativa,

13) $S_{1}$ es verdadera (V) si y sólo si la descripción que contiene describe un objeto ${ }^{1}$ que tiene la propiedad indicada en el predicado de $S_{1}$.

En cuanto a la falsedad Strawson ha reconocido (aunque no en el artículo que comentamos) que hay dos sentidos en que puede decirse que una oración predicativa es falsa. El primer sentido -el único reconocido en el artículo que comentamos- estaria caracterizado por:

14) $S_{1}$ es falsa $\left(F_{1}\right)$ sí y sólo si la descripción que contiene describe un objeto que no tiene la propiedad indicada en el predicado. (Uso aquí ' $F_{1}$ ' para distinguir este sentido de falsedad del próximo que identificaré con ' $\mathrm{F}_{2}{ }^{\prime}$ ).

En otro sentido,

15) $S_{1}$ es falsa $\left(F_{2}\right)$ si y sólo si no se da la condición para que $S_{1}$ sea verdadera $(\mathrm{V})$.

En las caracterizaciones anteriores de la noción de presuposición, la noción de falsedad que en ellas figura es Ia indicada en 14), es decir, $F_{1}$, pues es en este sentido que si $S_{2}$ no es $V$ (es decir, si la descripción contenida en $S_{1}$ no describe entidad alguna), $S_{1}$ no es verdadera $(V)$ ni es $\left(F_{1}\right)$, ya que tanto para la verdad (V) como para la falsedad $\left(F_{1}\right)$ de $S_{1}$ se requiere que la descripción que contiene describa una entidad, es decir que $S_{2}$ sea verdadera.

Pasemos ahora a analizar la noción de implicación. Un sentido razonable de esta noción es:

16) $S_{1}$ implica $_{2} S_{2}$ si y sólo si Si $S_{1}$ es $V$ entonces $S_{2}$ es $V$, o equivalentemente

17) $S_{1}$ implica ${ }_{2} S_{2}$ si y sólo si Si $S_{2}$ no es $V$ entonces $S_{1}$ no es $V$, pero como

18) $S_{1}$ no es $V$ si y sólo si $S_{1}$ es $F_{2}$.

Otra formulación equivalente de (16) es:

19) $S_{1}$ implica $_{2} S_{2}$ si y sólo si $S i S_{2}$ no es $V$ entonces $S_{1}$ es $F_{2}$.

He identificado a este sentido de implicación con el sub-índice ' 2 ' para indicar que puede ser caracterizada usando la segunda noción de falsedad $\left(\mathrm{F}_{2}\right)$.

Con este sentido de implicación resulta contradictorio afirmar que un enunciado presupone pero no implica a otro pues, resulta verdad que:

20) Si $S_{1}$ presupone $S_{2}$ netonces $S_{1}$ implica $S_{2}$. (Compárese 11a) y 16)).

1 "La descripción que contiene $S_{1}$ describe un objeto" abrevia de aquí en adelante a "Hay un y solo un objeto que tiene la propiedad indicada por el predicado contenido en la descripción contenida en $\mathbf{S}_{1}$ ". 
En este sentido cuando $S_{1}$ es una oración predicativa y $S_{2}$ la correspondiente oración existencial, resulta que:

21) $S_{1}$ presupone $S_{2}$ y $S_{1}$ implica $S_{2}$.

Pero hay otro sentido de implicación que probablemente sea el que Strawson ha tenido en cuenta cuando afirma que $S_{1}$ presupone $S_{2}$ pero no la implica.

Este sentido se obtiene partiendo de 19) y reemplazando ' $F_{2}$ ' por ' $F_{1}$ ' en ' $S_{1}$ es $F_{2}$ '. De este modo resulta:

22) $S_{1}$ implica ${ }_{1} S_{2}$ si y sólo si $S i S_{2}$ no es V. entonces $S_{1}$ es $F_{1}$.

Obsérvese que de acuerdo a los sentidos de ' $V$ ', ' $F_{1}$ ' y ' $F_{2}$ ' resultan:

23) Si $S_{1}$ es $F_{1}$ entonces $S_{1}$ no es $V$.

24) Si $S_{1}$ es $F_{1}$ entonces $S_{1}$ es $F_{2}$.

De 22), 24) y 19) o de 22), 17) y 23) se infiere:

25) Si $S_{1}$ implica $_{1} S_{2}$ entonces $S_{1}$ implica $_{2} S_{2}$.

Para obtener la relación conversa de 25) haría falta la conversa de 23).

26) Si $S_{1}$ no es $V$ entonces $S_{1}$ es $F_{1}$,

que de darse equivaldría al principio de tercer excluido con la noción de falsedad correspondiente a $F_{1}$, pues 26 ) equivale a:

27) $S_{1}$ es $V$ o $S_{1}$ es $F_{1}$.

Cuando Strawson sostiene que las oraciones predicativas pueden no ser verdaderas ni falsas, lo hace precisamente porque $F_{1}$ es su concepto de falsedad para tales oraciones. En este sentido, obviamente 26) y 27) son falsos y ello implica que la relación de implicación $n_{1}$ es más fuerte que la de implicación ${ }_{2}$, pues 25) es verdadera pero no su conversa.

Otra consecuencia de este sentido de falsedad es que si $S_{1}$ es una oración predicativa y $S_{2}$ su correspondiente existencial

28) Si $S_{2}$ no es $V$ entonces $S_{1}$ no es $F_{1}$ (consecuencia de 21) y 12)).

De esto se concluye para todo $S_{2}$ que no sea necesariamente verdadero, que:

29) $S_{1}$ no implica $S_{2}$.

Así se consigue el sentido de implicación en el que resulta que las oraciones predicativas presuponen pero no implican las existenciales.

En síntesis, puede resumirse la teoría de Strawson con referencia a la relación de las oraciones predicativas y las existenciales en las siguientes tesis:

TS 1$) S_{1}$ presupone $S_{2}$

TS 1.o) Si $S_{1}$ es V o $S_{1}$ es $F_{1}$ entonces $S_{2}$ es $V$

TS1.1) Si $S_{1}$ es $V$ entonces $S_{2}$ es $V\left(S_{1}\right.$ implica $\left._{2} S_{2}\right)$ (TS1 y 11)) (TS1 y 20))

TS1.2) Si $S_{1}$ es $F_{1}$ entonces $S_{2}$ es $V\left(T_{1}\right.$ y 11$)$ )

TS1.3) Si $S_{2}$ no es $V$ entonces $S_{1}$ no es $V$ y $S_{1}$ no es $F_{1}\left(T_{1}\right.$ y 12))

TS1.4) $S_{1}$ es $V$ o $S_{1}$ es $F_{2}$ (TS1.1 y 18))

TS2) $S_{1}$ no implica $S_{2}$ (cuando $S_{2}$ no es necesariamente V) (29)) 
De este modo se sustentan las tesis de presuposición y no implicación (TS1 y TS2), (4) e igualmente (3) que no siempre la oración predicativa es verdadera o falsa, ya que TS1.3 muestra la circunstancia en que no es verdadera ni falsa.

\section{ESQUEMA TEÓRICO DE LA TEORfA DE RUSSELL}

Cuando Russell analiza las oraciones predicativas y las existenciales y sus correspondientes relaciones lógicas, no lo hace como Strawson en un metalenguaje acerca de un lenguaje en el que ellas figuren, sino a través de la traducción de tales oraciones al lenguaje de Principia Mathematica introduciendo en el mismo, considerado como lenguaje objeto, las distinciones que él cree indispensables y que, como veremos, corresponden en forma directa y muy obvia a las hechas por Strawson.

Como es sabido Russell simboliza las oraciones predicativas por medio de! esquema

3o) $G(v x) F x$

que define por

31) (Ey) $[(x)(F x \equiv x=y) \cdot G y]$

de modo que en forma análoga a lo que se establece en 13) resulta

32) ' $G(\mathrm{I} x) \mathrm{Fx}^{\prime}$ ' es $\mathrm{V}$ si y sólo si la descripción que contiene ('( $\left.1 \mathrm{x}\right) \mathrm{Fx}$ ') describe un objeto que tiene la propiedad indicada por el predicado (' $G$ ').

Luego considera la situación que resulta cuando una oración predicativa figura dentro del contexto de otra oración. Detecta aquí una ambigüedad que en algunos escritos la presenta distinguiendo lo que llama figuraciones primarias de lo que llama figuraciones secundarias de una descripción y que en Principia Mathematica elimina mediante lo que llama el operador de alcance de una descripción. No expondré esta teoría en toda su extensión sino solamente para el caso en que la oración predicativa figure negada.

En este orden de cosas, cuando un enunciado como 30 ) es negado hay dos sentidos en que puede considerarse su negación: (1) el que corresponde a lo que Russell llama la figuración secundaria, que simbolizaremos con

33) $\sim \mathrm{G}(1 \mathrm{x}) \mathrm{Fx}$

y que es verdad si y sólo si 31$)$ no lo es, es decir cuando se da o sea

34. $\sim(E y)[(x)(F x \equiv x=y) \cdot G y]$.

35) ' $G(2 x) F x$ ' es $V$ si y sólo si ' $G$ ( $3 x) F x$ ' es $F_{2}$ en la terminología que usamos al exponer a Strawson. (2) El segundo sentido corresponde a lo que sería la figuración primaria frente a la negación, que simbolizaremos por 
36) $7 G(2 x) F x$

que es verdad si y sólo si se da

37) (Ey) $[(\mathrm{x})(\mathrm{Fx} \equiv \mathrm{x}=\mathrm{y}) \cdot \sim \mathrm{Gy}]$

que en forma análuga a lo que se indica en 14 ) resulta verdadera si y sólo si la descripción ' $(\mathrm{I}) \mathrm{Fx}$ ' describe un objeto que no tiene la propiedad indicada en el predicado ' $G$ ', es decir

38) ' $7 \mathrm{G}(1 \mathrm{x}) \mathrm{Fx}$ ' es $V$ si y sólo si ' $G(1 \mathrm{x}) \mathrm{Fx}$ ' es $F_{1}$ en la terminología usada para exponer a Strawson.

De este modo se ve con claridad que los dos sentidos de falsedad que distingue Strawson corresponden perfectamente a las dos formas de negación (primaria y secundaria) que encontramos en Russell. Aunque a decir verdad Strawson, en su artículo originario, no parece haberse percatado de estos dos sentidos en que puede usarse la noción de falsedad, manejando sólo el que llamamos $F_{1}$. Tampoco parece haberse dado cuenta de la importancia de las dos formas de figuración que analiza Russell, por cuanto ni siquiera las menciona.

Las oraciones existenciales Russell las simboliza por

39) $\exists !(1 \mathbf{x}) \mathbf{F x}$

que define por

40) $(\mathrm{Ey})(\mathrm{x})(\mathrm{Fx} \equiv \mathrm{x}=\mathrm{y})$

$y$ en forma análoga a lo indicado en ${ }^{\prime \prime} 5^{\prime \prime}$ ).

$41)$ ' $\exists$ ! ( $3 x) F x$ ' es $V$ si y sólo si hay un objeto y sólo uno que '( $1 \mathbf{x}) \mathrm{Fx}^{\prime}$ describe.

De este modo expondré, con la terminología indicada, algunas de las leyes características de la lógica de Russell para las proposiciones que contienen descripciones.

42) $[G(1 x) F x$ v $7 G(1 x) F x] \supset \exists !(1 x) F x$.

Que en virtud de 32$\left.), 3^{8}\right), 4^{1}$ ), y traducciones obvias de los conectivos proposicionales es equivalente a

43) Si ' $G(1 x) F x$ ' es $V$ o ' $G(1 x) F x$ ' es $F_{1}$ entonces ' $\exists$ ! ( $\left.1 x\right) F x$ ' es $V$ que si se toma $S_{1}$ como nombre de ' $G(1 \times) F x$ ' y $S_{2}$ de ' $\exists$ ! $(1 \times) F x$ ' no es más que TS 1.o) y que a su vez es equivalente a

44) ' $G$. ( 1 x ) Fx' presupone ' $\exists$ ! ( $1 x) F x$ '

que no es más que TS1) la tesis fundamental de Strawson.

Otros teoremas, consecuencias obvias de $4^{2}$ ), son

45) $G(1 x) F x \supset \exists l(1 x) F x$

46) $7 \mathrm{G}(\mathrm{i} x) \mathrm{Fx} \supset \exists !(1 \mathrm{x}) \mathrm{Fx}$

47) $\sim \exists$ ! (1 $x) F x \supset[\sim G(1 x) F x \cdot \sim 7 G(1 x) F x]$

$\left.4^{\prime}\right) \mathrm{G}(1 \mathrm{x}) \mathrm{Fx} \mathrm{V} \sim \mathrm{G}(1 \mathrm{x}) \mathrm{Fx}$

que pueden formularse, por lo indicado anteriormente

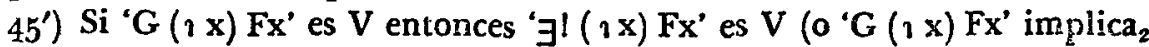
' $\exists !(3 \times \mathrm{Fx}$ ') 
$4^{6}$ ') Si ' $G(2 x)$ Fx' es $F_{1}$ entonces ' $\exists$ ! ( $\left.1 x\right)$ Fx' es $V$

47') Si ' $\exists !(1 x) F x^{\prime}$ no es $V$ entonces ' $G(1 x) F x$ ' no es $V$ y ' $G(2 x) F x$ ' no es $F_{1}$

$\left.4^{8}\right)$ ' $G(1 x) F x$ ' es $V_{0}$ ' $G(1 x) F x$ ' es $F_{2}$

Como es claro éstas corresponden a TS1.1), TS1.2), TS1.3), y TS1.4).

En cambio, no son tesis de Principia

49) $G(1 \times) F x$ v $\neg G(1 x) F x$

59) $\sim \exists$ ! (1x) F $\supset \supset 7 \mathrm{G}(1 \mathrm{x}) \mathrm{Fx}$.

La primera (49)), corresponde al rechazo de Strawson del principio de tercero excluido con la noción de $\mathrm{F}_{1}$, $\mathrm{O}$ sea de

$\left.49^{\prime}\right)$ ' $G(1 x) F x$ ' es $V$ o ' $G(1 x) F x$ ' es $F_{1}$

la segunda (50)), al rechazo de la implicación ${ }_{1}$ indicado en TS2), o sea, el rechazo de

$\left.5^{\circ}\right)$ ' $\exists$ ! ( $1 x$ ) Fx' implica ' $G(2 x) F x$ '.

El único caso en que 49 ) y 50 ) son tesis de Principia es cuando el enunciado ' $\exists !(1 x)$ Fx' es necesario, como, por ejemplo, si fuera ' $\exists$ ! $(2 x)(x=z)$ ', ya que es teorema de Principia Mathematica. ' $(z) \exists !(\mathbf{x})(x=2)^{\prime}$, pero la misma excepción debe hacerse en la teoría de Strawson.

En síntesis, la teoria de Strawson no difiere de la de Russell en la medida en que tocan temas comunes. Lo que Russell expresa con la figuración primaria de una descripción en el contexto de una negación (" en nuestro simbolismo simplificado) es lo que Strawson expresa metalingüisticamente con su noción de falsedad ${ }_{1}\left(F_{1}\right)$ y lo que Russell expresa con la figuración secundaria es lo que Strawson expresa con la noción de falsedad ${ }_{2}\left(F_{2}\right)$, el resto es sólo diferencia de formulación, sin más alcance que un cambio de terminologia.

De este modo creo haber justificado que Strawson no ha mostrado ninguna falla en la teoría de Russell, por cuanto la suya se limita a reproducir, con terminología diferente, parte de la teoria de Russell, que por cierto, es mucho más amplia que la de Strawson.

Hay otro sentido de 4), el rechazo de Strawson de que cuando se afirma "El rey de Francia es sabio" parte de lo que se estaría afirmando es que en la actualidad existe un rey de Fracia y sólo uno.

Esto depende de una teoria acerca de los actos lingüisticos de afirmar una oración (en este caso predicativa) y no del análisis lógico de las oraciones objeto de la afirmación, en otras palabras, de lo que suele llamarse una lógica de la afirmación. Simbolicemos con

51) A X S

el enunciado que describe la afirmación que un individuo $x$ hace de (o con) la oración S. La pregunta que puede aquí formularse es: Cuando

52) $S$ implica lógicamente a $S^{\prime}$ ¿se sigue de ello que 


\section{3) $\mathrm{A} \times \mathrm{S}^{\prime}$ ?}

Hay sentidos de la expresión 'afirmar' en los que no es verdad que cuando alguien afirma algo necesariamente afirma todo lo implicado por su afirmación, aunque normalmente no se excluya que algo de lo implicado por lo afirmado es igualmente afirmado; y hay sentidos en los que todo lo implicado por lo afirmado es igualmente afirmado.

Cuando Frege afirmó las tesis de su teoría lógica cafirmó una contradicción? ¿Debería concluirse tal cosa de la demostración de Russell de que de ellas se implicaba una contradicción?

Este es un tema que no deseo discutir aquí pues no tiene nada que ver con el problema de la teoría lógica de los enunciados que contienen descripciones, sino con lo que, como indiqué, sería una lógica de la afirmación, o una teoría de los actos lingǘsticos de afirmar. Cualquiera sea el resultado de tal teoría, en nada incidirá en lo que pueda responderse frente a las preguntas referidas a las relaciones lógicas (Verdad, Falsedad, Implicación, Presuposición, etc.) entre los enunciados. A la inversa, es sólo cuando se tiene una logica clara para las oraciones que pueden empezarse a plantear con claridad los problemas relacionados con el tema de la afirmción.

Una última aclaración: muchas veces una descripción es usada en un enunciado predicativo sólo para identificar el objeto motivo de la predicación, de modo que para la verdad de tal enunciado no se requiere que el objeto identificado tenga la propiedad correspondiente al predicado contenido en la descripción. En tales casos un enunciado predicativo no implica en ningún sentido el enunciado existencial, pero en tales casos tampoco lo presupone. Obviamente no es para el caso de descripciones así usadas, que Strawson ha desarrollado su teoría, pues al aceptar 2) supone que la verdad del enunciado existencial es condición necesaria para la verdad del enunciado predicativo y entonces excluye tal situación.

Facultad de Derecho y Ciencias Soctales UNIVERSidAd DE Buenos AIRES

Carlos E. Alchourrón 